CRC do not satisfy criteria for syndromes such as HNPCC, and fall into a "moderate risk" category. The reported polyp burden in this group is varied, and the optimum screening regimen is controversial. Our aims were (1) to evaluate the polyp yield at screening colonoscopy in a "moderate risk" group (above average, non-HNPCC) in the setting of a family-screening clinic, (2) to compare polyp yield on 2 nd screening colonoscopy between patients with and without adenomas on 1st screening colonoscopy, (3) to evaluate the potential for longer screening intervals for patients with no adenomas on 1 st screening colonoscopy.

Methods Family cancer history questionnaires were used to generate family pedigrees and identify "moderate risk" individuals using defined criteria. Adenoma yield on initial colonoscopy was evaluated, and comparisons were made between males \& females, and subjects older \& younger than 50 yrs. Advanced adenomas (AA) were defined as adenomas $\geq 10 \mathrm{~mm}$, with high-grade dysplasia, or with a villous component. In patients who had $>1$ colonoscopy, adenoma yield on 2 nd colonoscopy was compared between patients with and without adenomas on initial colonoscopy.

Results From a cohort of 2008 individuals in a high-risk familyscreening clinic, 971 (48\%) have been assigned a "moderate risk" category. Complete data were available for screening colonoscopies in 236 of these; 99 male, 137 female. On initial screening colonoscopy, 17/236 (7\%) had AA, and a further 37/236 (16\%) had simple adenomas (SA), (total polyp yield $23 \%$ ). Polyp yield was higher in males ( $8 \% \mathrm{AA}, 18 \% \mathrm{SA})$ vs females (7\% AA, 14\% SA), and in the $>50$ yrs $(13 \%$ AA, $20 \%$ SA) vs $<50$ yrs ( $3 \%$ AA, $13 \%$ SA). More than 1 screening colonoscopy was carried out in $127 / 236$ (54\%). Of the $30 / 127(24 \%)$ who had an adenoma on initial colonoscopy, 4/30 $(13 \%)$ had AA, and a further $7 / 30(23 \%)$ had SA on $2^{\text {nd }}$ colonoscopy (mean interval to $\mathrm{f} / \mathrm{u} 3.62 \mathrm{yrs}$ ). In the cohort without adenomas at initial screening; $97 / 127$ (76\%), only $1 / 97$ (1\%) had an AA, and 10/97 (10\%) had SA on 2nd colonoscopy (mean interval $4.6 \mathrm{yrs}$ ).

Conclusion In this moderate risk group the polyp yield is highest in males, and those $>50$ yrs. Adenoma at initial colonoscopy was predictive of adenoma detection at 2 nd colonosocopy. In contrast, for individuals without adenomas at initial screening, a very low adenoma yield was observed at follow-up screening. Consequently, within this "moderate risk" cohort, the data supports the adoption of differing screening protocols depending on age, gender, and adenoma yield on initial colonoscopy.

Competing interests None declared.

\section{PTU-227 IMPROVING EFFICIENCY IN CAPSULE ENDOSCOPY: CAN READING TIMES BE REDUCED WITHOUT SACRIFICING DIAGNOSTIC ACCURACY? A SELF-ASSESSMIENT}

doi:10.1136/gutjnl-2012-302514c.227

M Nakamura, ${ }^{*}$ A Murino, A Fitzpatrick, C Fraser. The Wolfson Unit for Endoscopy, St Mark's Hospital and Academic Institute, Imperial College, London, UK

Introduction Capsule endoscopy $(\mathrm{CE})$ is a time consuming procedure. The RAPID 7 Access reading software (Given Imaging Ltd) has three patterns of view modes (VM) (one view, VM1; double views, VM2; quadruple views, VM4) and an adjustable frame rate (AFR) from 5 to $40 \mathrm{fps}$. The appropriate settings for VM and AFR depend on capsule endoscopist's experience, and a consensus has not been achieved yet. The aim of this study was to investigate how different VM's and AFR's could influence diagnostic accuracy.

Methods An entire capsule endoscopy procedure consisting of 27 small bowel angioectasias was selected from our database. This was read by a single expert capsule endoscopist repeatedly using 11 different randomised combinations of VM and AFR (1, 2 and 4 VM $\times 10,15,25$ and $40 \mathrm{fps}$ ). Reading times and number of angioectasias detected for each combination were recorded and then compared.
Results The small bowel transit time was 321 min. Mean reading times (all VM's) at 10, 15, 25 and 40 fps respectively were 34, 22, 14 and $10 \mathrm{~min}$. Considering $10 \mathrm{fps}$ as the gold standard for reading, the reduction in reading time at 15, 25 and $40 \mathrm{fps}$ was 33\%, 60\% and $70 \%$ respectively. No significant differences were noticed in reading times between VM's at the same AFR. A mean of 23, 16, 7 and 6 angioectasias were detected at 10,15, 25 and 40 fps respectively (all VM's combined). Diagnostic accuracy at 25 and $40 \mathrm{fps}$ was significantly lower than $10 \mathrm{fps}(\mathrm{p}=0.04,0.01)$. The mean numbers of detected angioectasias according to VM were 14, 17 and 16 for VM1, VM2 and VM4 respectively. The lowest number of angioectasias (5) was detected using VM2 $\times 40 \mathrm{fps}$. The highest number of angioectasias $(25)$ was detected using VM2 $\times 10 \mathrm{fps}$ and VM4 $\times$ $10 \mathrm{fps}$. Using VM2 $\times 15 \mathrm{fps}, 18$ angioectasias were detected, meaning that diagnostic accuracy was reduced to $72 \%$ (compared with VM2 $\times 10 \mathrm{fps}$ ), although the reading time decreased by $33 \%$. Conclusion Our findings suggest that the highest diagnostic accuracy was achieved with VM $2 \times 10 \mathrm{fps}$ or VM $4 \times 10 \mathrm{fps}$. The AFR influences both diagnostic accuracy and reading time. As the AFR increases, reading times are reduced but this is associated with a reduction in diagnostic accuracy and a concomitant increase in miss rates. Capsule endoscopists need to be aware of this phenomenon.

Competing interests None declared.

\section{PTU-228 PREDICTING DIFFICULT COLONOSCOPY USING THE ST MARK'S DIFFICULT COLONOSCOPY SCORING SYSTEM: A PILOT STUDY}

doi:10.1136/gutjnl-2012-302514c.228

M Nakamura, * A Murino, E Despott, N Suzuki, L Bourikas, R Man, C Fraser. The Wolfson Unit for Endoscopy, St Mark's Hospital and Academic Institute, Imperial College, London, UK

Introduction Colonoscopy can sometimes be difficult. This may be due to a number of factors such as age, gender, increased colon length, waist/hip ratio $<1, \mathrm{BMI}<22$, abdominopelvic surgery and a history of constipation. Colonoscopists tend to develop their own strategies based on their personal experience and the availability of specialised equipment. A scoring system based on these factors could be a useful predictor of difficult colonoscopy with the advantage that such a score could be calculated prior to the procedure. We therefore developed an evidence based difficult colonoscopy score (DCS), incorporating factors associated with difficult colonoscopy. The aim of this study was to validate the reliability of the proposed St Mark's DCS evaluating the relationship between each factor and caecal incubation time.

Methods Patients referred for routine colonoscopy were recruited. 30 patients were prospectively selected. Each patient was screened using a questionnaire. Colonoscopies were started with an adult colonoscope, but if needed, alternative options such as a paediatric colonoscope or real time magnetic imager were made available on request.

Results The overall caecal incubation rate was 97\% (29/30). One patient was excluded due to a colonic stricture. The median DCS was 3 (range 0-6). Median insertion time was 8 min (range 3-23). In three patients colonoscopists changed to an alternative option during colonoscopy. There was a significant correlation between the DCS and insertion time ( $\mathrm{r}=0.511, \mathrm{p}=0.005$, Pearson's correlation coefficient). Moreover, if the DCS was five or more, caecal intubation time was $>15$ min suggesting a strong correlation. The significant factors by univariate analysis influencing a caecal intubation time of more than $15 \mathrm{~min}$ were "Waist/hip ratio $<1$ and/or $\mathrm{BMI}<22$ ", "over 60 years old" and "Constipation". Multivariate analysis suggested the most significant factor for difficult colonoscopy was a history of constipation. 